\title{
IoT based System for Heart Rate Monitoring
}

\author{
Sahana S Khamitkar \\ M.Tech (CSE) \\ Department of Studies in Computer Science and \\ Engineering \\ University B.D.T College of Engineering, \\ Davanagere -577004, Karnataka, India \\ ( A Constituent College of Visvesvaraya Technological \\ University, Belagavi )
}

\begin{abstract}
The Heart Rate Monitoring system is developed using IOT technology with an objective of detecting the heart beat of the patient in order to monitor the risk of heart attack and also the regular checkup. Body health monitoring is very important to us to make sure our health is in excellent condition. One of the vital parameter for this device under consideration is the heart rate (HR). In this project we describe the design of low cost heart rate monitoring device from fingertips based on the Bluetooth technology. The entire system is comprised of several parts such as Heart Rate module, Android application and Bluetooth module. The Heart Rate (HR) module picks up heart rate signal by a noninvasive technique (Photoplethysmography) from the subject (patients) and sends it (signal) wirelessly to computer or android application using Bluetooth module. This system can be embraced and combined as a part of telemedicine constituent. The data received from heart rate module can be saved and viewed for further medical usage. The result from this device prototype can be utilized for various clinical investigations, indeed these Bluetooth's signal can be transmitted between 15 to 20 meters radius.
\end{abstract}

Keywords: Heart Rate, IoT, Photoplethysmography, Bluetooth

\section{INTRODUCTION}

The heart is one of the most important organs in the human body. It acts as a pump for circulating oxygen and blood throughout the body, thus keeping the functionality of the body intact. A heartbeat can be defined as a two-part pumping action of the heart which occurs for almost a second. It is produced due to the contraction of the heart. When blood collects in upper chambers, the SA(Sino Atrial) node sends out an electrical signal which in turn causes the atria to contract. This contraction then pushes the blood through tricuspid and the mitral valves; this phase of the pumping system is called diastole. The next phase begins when the ventricles are completely filled with blood. The electrical signals generating from SA node reach the ventricle and cause them to contract. In today's scenario, health problems related to heart are very common. Heart diseases are one of the most important causes of death among men and women. It claims approximately 1 million deaths every year. Heart rate is a critical parameter in the functioning of the heart. Therefore heart rate monitoring is crucial in the study of heart performance and thereby maintaining heart health.

\author{
Prof. Mohammed Rafi \\ Department of Studies in Computer Science and \\ Engineering \\ University B.D.T College of Engineering, \\ Davanagere -577004, Karnataka, India \\ ( A Constituent College of Visvesvaraya Technological \\ University, Belagavi )
}

This paper proposes a heart rate monitoring detection system using IoT. Nowadays treatment of most of the heart-related diseases requires continuous as well as long term monitoring. IoT is very useful in this aspect as it replaces the conventional monitoring systems with a more efficient scheme, by providing critical information regarding the condition of the patient accessible by the doctor. In addition, the nurses or the duty doctor available at the hospital can monitor the heart rate of the patient in the serial monitor through the real-time monitoring system.

\section{PHOTOPLETHYSMOGRAPHY:}

Photoplethysmography (PPG) is a low cost optical technique that is able to detect volumetric changes in blood flowing through capillaries from the skin surface. Photoplethysmography was developed in the late $1800 \mathrm{~s}$ where scientists observed real-time blood flow using light bulbs. It was in the late 1930s that the term "photoplethysmography" was coined by scientists. With advancement in technology, PPG developments now focus on consumer applications using wearable devices. These wearable devices are usually connected to a peripheral device to interpret the results obtained. Today, those peripheral devices have been replaced by smart phones to deliver data to consumers in a user friendly manner. Interfacing with smart phones is done using Bluetooth technology. Bluetooth is a low power consuming wireless communication technology that enables users to connect, transfer, and receive data over the air between two compatible devices.
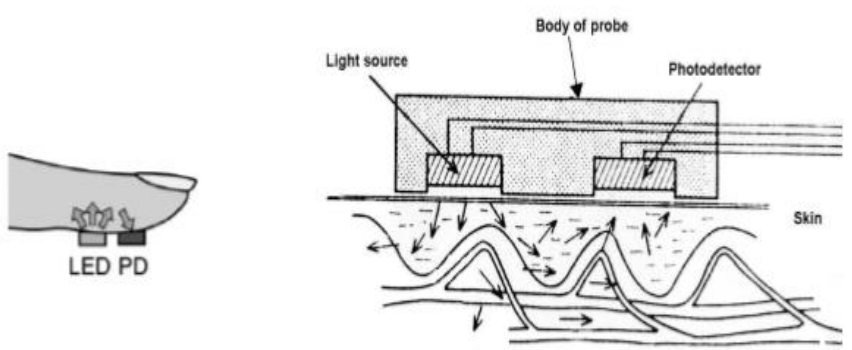

Photoplethysmography is a derivative of plethysmography techinique, where in a simple optical setup can help detect volume changes in peripheral blood circulation. This technique is a non-invasive method since it makes measurements at the skin surface. The technology uses 
optoelectronic components, such as a red or near infrared light source, to illuminate skin and a photo detector to observe the variations in light intensity within the observed area. Usually, a red or near infrared source of light is used to illuminate skin. This light then travels through tissues and is absorbed by pigments, bones, and blood. The PPG sensors optically observe changes in blood flow volume by detecting changes in light intensity.

\section{LITERATURE SURVEY}

1. A microcontroller based automatic heart rate counting system from fingertip Mamun AL, Ahmed N, ALQahtani (JATIT)Journal OF Theory and Applied technology ISSN 1992-8645 : In this research paper heartrate signals were collected from finger or ears using IR TX-RX (Infrared Transmitter and Receiver pair) module which was amplified in order to convert them to an observable scale. A low pass filter was used to filter inherent noise. These signals were counted by a microcontroller module (ATmega8L) and displayed on the LCD. Microcontroller is programmed with an algorithm to run the proposed heart rate counting system. The results obtained using this process when compared to those obtained from the manual test involving counting of heart rate was found satisfactory. The proposed system is applicable for family, hospital, community medical treatment, sports healthcare and other medical purposes. Also, fit for the adults and the pediatrics. However, this method in the developed system needs further investigation and need more functionality, which may be useful to consider advance in future research .

\section{Heart beat Sensing and Heart Attack Detection Using} internet of things: IOT Aboobacker sidheeque, Arith Kumar, K. Sathish,(IJESCE) International Journal Of Engineering Science and Computing, April 2007 : In this research paper implementation of heartbeat monitoring and Heart attack detection system using Internet of things is shown. These days we saw increased number of heart disease and heart attack. The sensor is interfaced to a microcontroller that allows checking heart rate readings ad transmitting them over internet. The user may Set the level of heart beat limit. After setting these limits, the system starts monitoring and as soon as patient heart beat goes above a certain limit, the system sends an alert to the controller which then transmits this over the internet and alerts the doctors as well as concerned users. Also, the system alerts for lower heartbeats. Whenever the user logs on for monitoring, the system also displays the live heart rate of the patient. Thus, concerned patients may monitor heart rate as well get an alert of heart attack to the patient immediately from anywhere and the person can be saved on time.

3. A Heartbeat and Temperature Measuring System for Remote Health Monitoring using Wireless Body Area Network Mohammad Wajih Alam , Tanin Sultana and Mohammad Sami Alam International Journal of BioScience and Bio-Technology Vol.8, No.1 (2016) : In this research paper, the design and development of a microcontroller based heartbeat and body temperature monitor using fingertip and temperature sensor is shown. The device involves use of optical technology to detect the flow of blood through the finger and offers the advantage of portability over conventional recording systems. Wireless body area network based remote patient monitoring systems have been presented with numerous problems including efficient data extraction and dynamic tuning of data to preserve the quality of data transmission. Evaluation of the device on real signals shows accuracy in heartbeat measurement, even under intense physical activity. This paper presents these challenges as well as solution to these problems by proposing an architecture which allows a network to be formed between the patient and doctor in order to enable remote monitoring of patient by analyzing the data of patient. The device consists of sensors which are used to measure heartbeat as well as body temperature of a patient and it is controlled by a central unit. The readings from these sensors are further processed and sent via GSM module to a remote location where it is displayed on cell phone. The optical heartbeat sensor counts the heartbeat per minute and Temperature sensor measures the temperature from the body and both the measured data are sent to a receiving end utilizing wireless technology where the data is displayed in a cell phone for further processing and patient care. This device is shown superior in comparison to traditional systems.

4. Heartbeat Monitoring Alert via SMS 2009 IEEE Symposium on Industrial Electronics and Applications October 4-6, 2009, Kuala Lumpur, Malaysia. Warsuzarina Mat Jubadi, Siti Faridatul Aisyah Mohd Sahak Dept. of Electronics Engineering University Tun Hussein Onn Malaysia Batu Pahat, Johor, Malaysia : In this research paper, it is shown that the heart rate can be measured by monitoring one's pulse using specialized medical devices such as an electrocardiograph (ECG), portable wrist strap watch, or any other commercial heart rate monitors. Despite of its accuracy, somehow it is costly, involve many clinical settings and patient must be attended by medical experts for continuous monitoring. For a patient whom already diagnosed with fatal heart disease, their heart rate condition has to be monitored continuously. This paper proposed an alert system that able to monitor the heart beat rate condition of patient. The heart beat rate is detected using photo plethysmograph (PPG) technique. This signal is processed using PIC16F87 microcontroller to determine the heart beat rate per minute. Then, it sends SMS alert to the mobile phone of medical experts or patient's family members, or their relatives via SMS. This will also alert the family members to quickly attend the patients.

\section{METHODOLOGY}

In this system we use the pulse sensor with Arduino Uno and Bluetooth HC-05 module, the pulse sensor is placed on the finger and it measures the heart rate and then sends the heart rate to android mobile application via 
Bluetooth. Early recognition of the disease is very vital in preventing more complications in the future.

There is three cases in which the heart rate is displayed:

1) Low Pulse Rate: The low pulse rate is displayed when the heart rate per BPM(Beats per minute) is $>40$ and $<60$. The low pulse rate may lead to medical complications this indicates that the patient needs the doctor's help(ex: Low BP)

2) Normal Pulse Rate: The normal pulse rate range's is between $>60$ and $<100$ which indicates that the patient has the normal range pulse rate with no complication.

3) High Pulse Rate: The high pulse rate is between $>100$ and $<150$ which indicates the patient has the high pulse range that could result in the heart related diseases(ex: High Blood Pressure)

The above readings are displayed in the mobile based application known as Blynk via Bluetooth.
BLOCK DIAGRAM

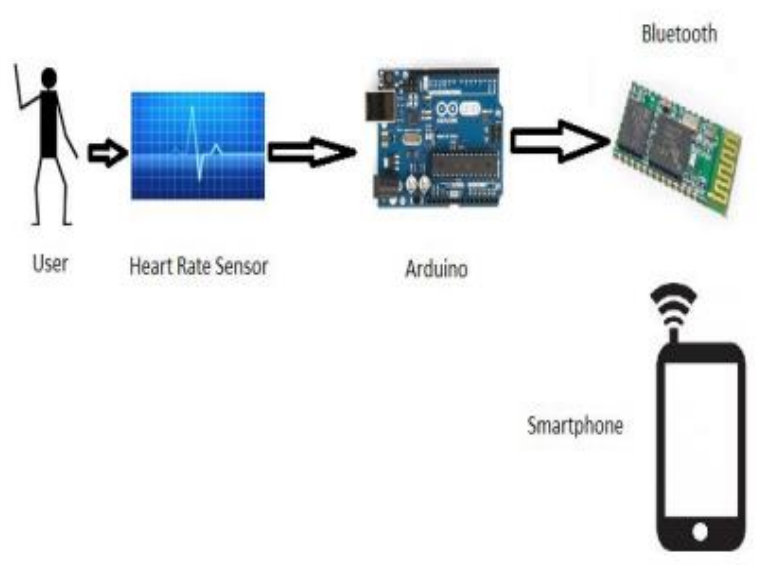

AVERAGE HAERT RATE

\begin{tabular}{l|l}
\multicolumn{1}{c|}{ Age } & Average Heart Rate \\
\hline Newborn Baby & 140 \\
\hline 7 year & $85-90$ \\
\hline 14 year & $80-85$ \\
\hline Adult & $70-80$ \\
\hline Athletes & $60-100$ \\
\hline
\end{tabular}

CIRCUIT IMPLIMENTATION

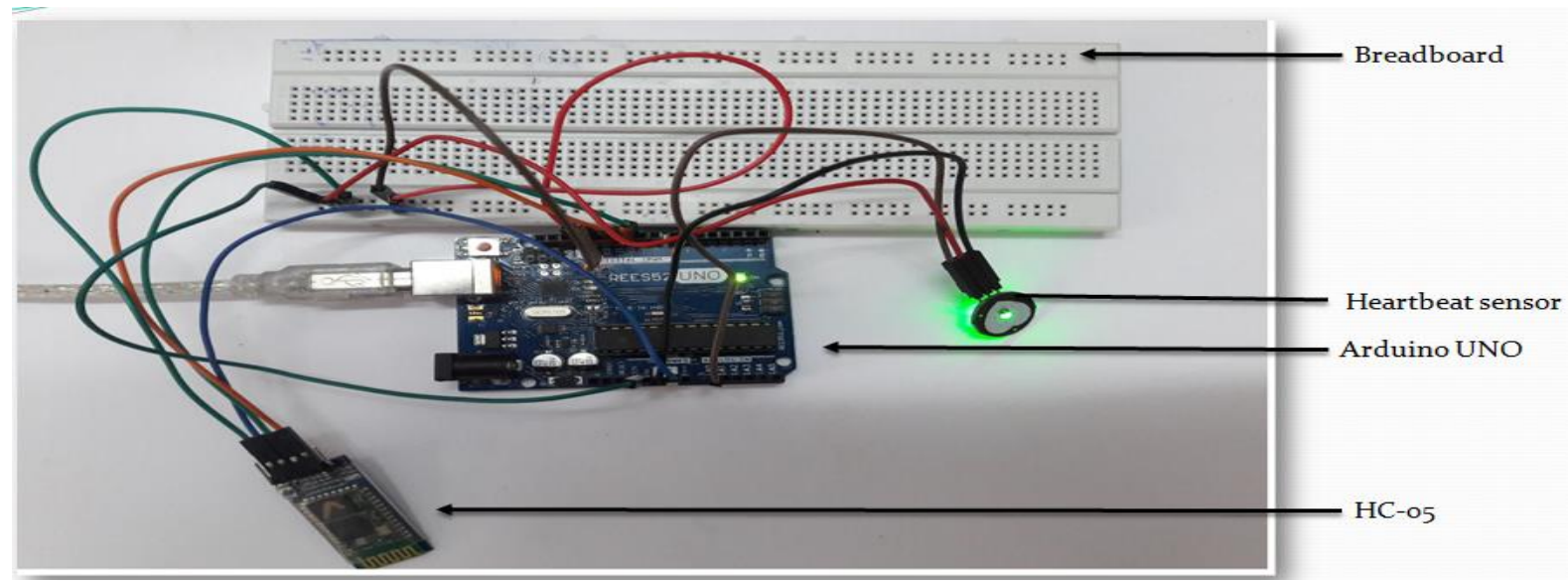

Above diagram represents the circuit connections of the components which includes Arduino Uno, Pulse sensor, Bluetooth module which in turn is connected to the computer. 


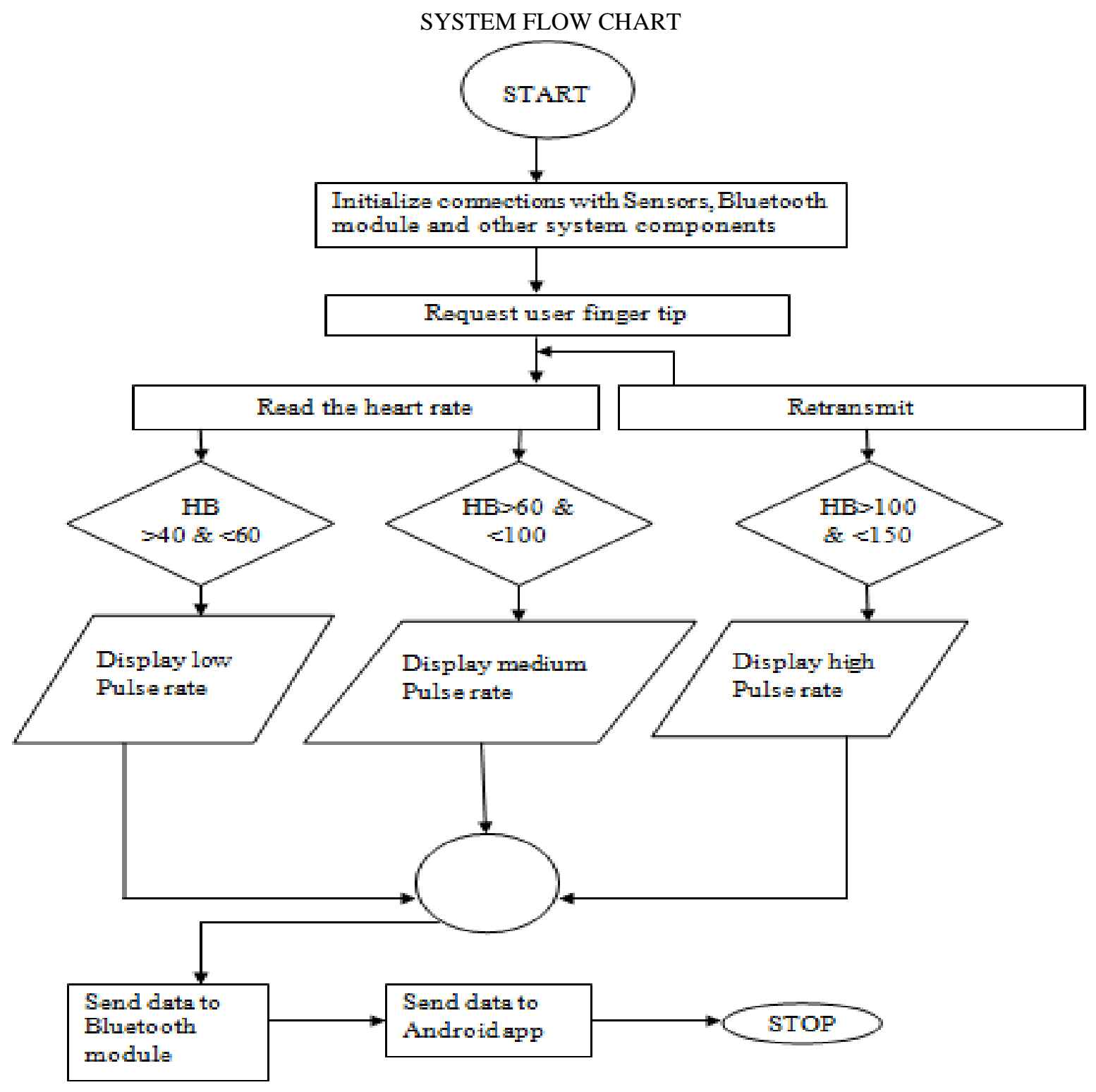

\section{SOFTWARE REQUIREMENTS}

1) Arduino IDE

2) Blynk App

ARDUINO IDE: Arduino IDE tool (software) is an open source environment where we can write a code and upload it on to UNO board. It runs on windows Mac OS and Linux OS. Is the required software environment to program the Arduino by writing a code and upload it to The Arduino. It also outputs the results for analysis using both serial monitor and serial plotter.

BLYNK APP: Blynk is a platform with iOS and Android apps to control Arduino, Raspberry Pi and the likes over the Internet. Blynk was designed for the Internet of Things.
It can control hardware remotely, it can display sensor data, it can store data, visualize it and do many other cool things.

There are three major components in the Platform:

1) BLYNK APP: it allows to create amazing interfaces for projects using various widgets we provide.

2) BLYNK SERVER: responsible for all the communications between the Smartphone and hardware. You can use our Blynk Cloud or run your private Blynk server locally. It is a opensource, could easily handle thousands of devices and can even be launched on a Raspberry Pi.

3) BLYNK LIBRARIES : for all the popular hardware platforms - enable communication with the server and process all the incoming and out coming commands. 


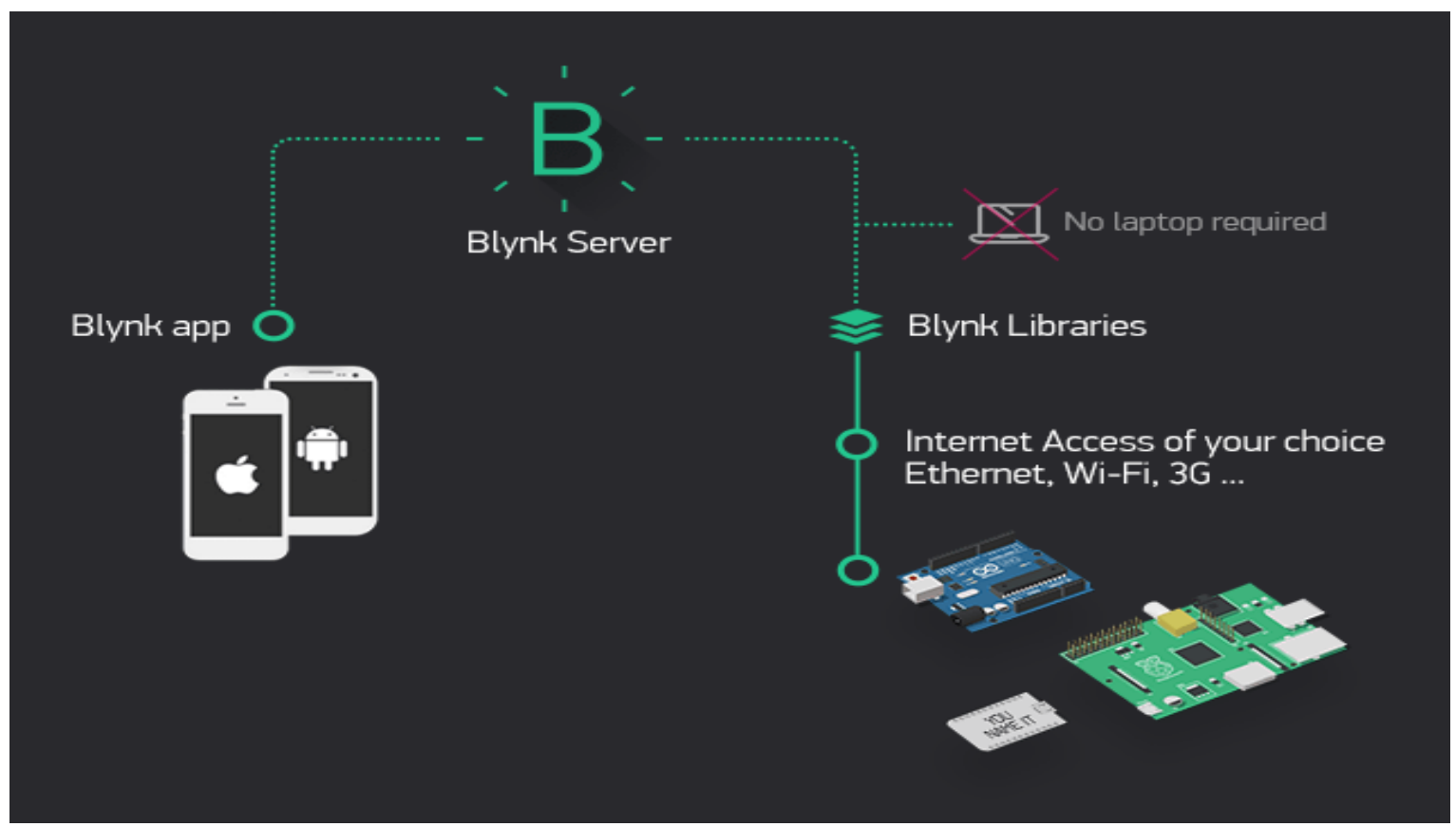

\section{FEATURES OF BLYNK}

1) Similar API \& UI for all supported hardware \& devices.

2) Connection to the cloud using $\mathrm{WiFi}$, Bluetooth and BLE(Bluetooth low energy).

3) Set of easy-to-use Widgets

4) Direct pin manipulation with no code writing

5) Easy to integrate and add new functionality using virtual pins

6) History data monitoring via Super Chart widget

7) Device-to-Device communication using Bridge Widget

8) Sending emails, tweets, push notifications, etc.

\section{HARDWARE REQUIREMENTS}

1) Arduino Uno

2) Pulse sensor

3) HC-05 Bluetooth

4) Jumper wires

5) Bread board

6) USB cable

7) Android Mobile device

ADRUINO UNO: Arduino UNO is micro-controller based on Atmega328, having 14 digital In/Out pins of which 6 are for PWM output, 6 are for analog input. Operates at $16 \mathrm{MHz}$, with a USB, Power jack, Reset button.

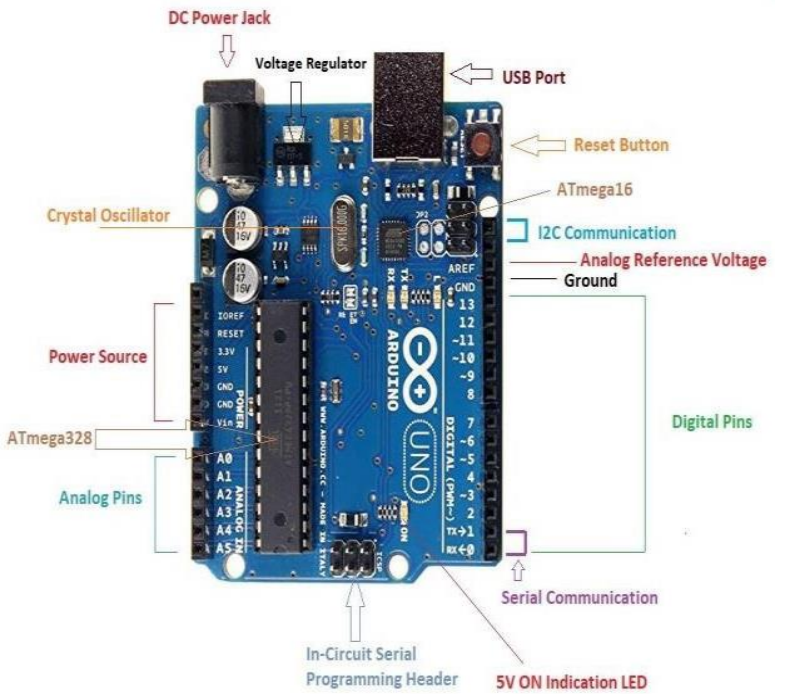

PULSE SENSOR: It is an Open Source heart rate monitor which considered as a PPG device used to monitor the non-invasive heart rate. It measures the realtime heart beats and calculates BPM with the aid of algorithms implemented by Arduino. The normal operating voltage is $+5 \mathrm{~V}$ or $+3.3 \mathrm{~V}$ and current consumption of $4 \mathrm{~mA}$. The sensor has two sides, one side consists of an LED with an ambient light sensor and the other side contains circuitry which amplifies the signals and filters the noise. 


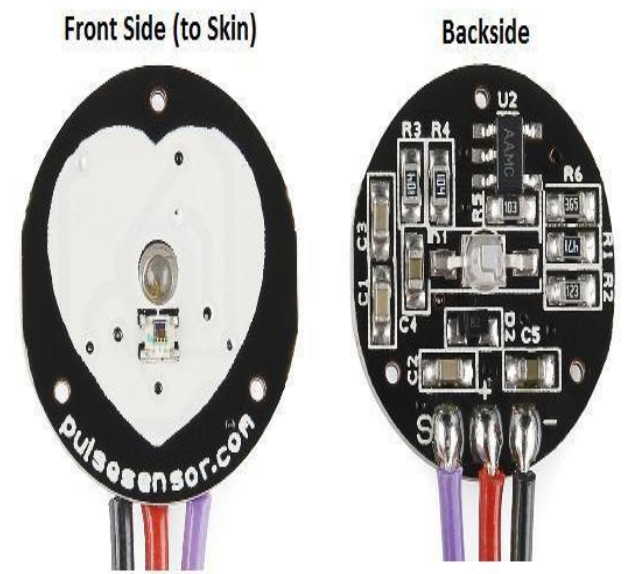

HC-05 BLUETOOTH: HC-05 is a serial port Protocol(SPP) designed for wireless serial connection setup. It was chosen for its ability to simplify the circuit design Send measured data to android application and due to its compatibility with the Arduino. In addition, it was chosen over HC-06 module because it has the ability to operate as both as master and slave modules rather than just a slave in the case of HC-06. TheHC-05 has six Pins, four of them are programmable input/output lines. While the three others, one is GND and the other is for VCC.

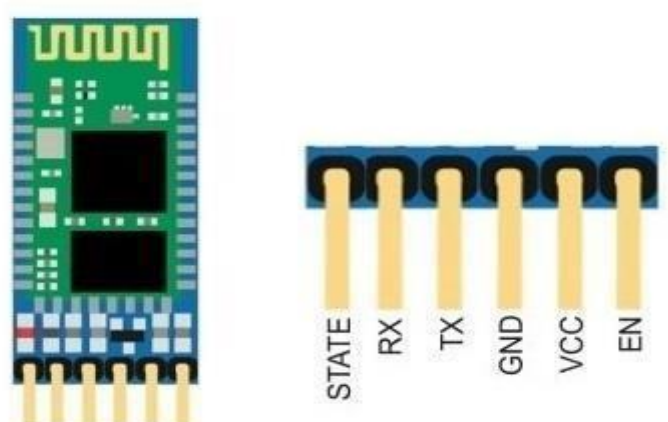

JUMPER WIRES: Jumper wires are simply wires that have connector pins at each end, allowing them to be used to connect two points to each other without soldering. Jumper wires are typically used with breadboards and other prototyping tools in order to make it easy to change a circuit as needed.

BREAD BOARD: A breadboard is a solder less device for temporary prototype with electronics and test circuit designs. The breadboard has strips of the metal underneath the board and connect the holes on the top of the board.

USB cable: USB cable is used to connect computer to the Arduino UNO board.

ANDROID MOBILE DEVICE: Smartphone enables technologies such as built in Bluetooth, near field communication (NFC), radio frequency Tracking(RFID) etc.

\section{RESULTS SNAPSHOOT}

Results on serial Monitor:

Below Snapshot represents the output that is the pulse rate of the person in the serial monitor of Arduino IDE.

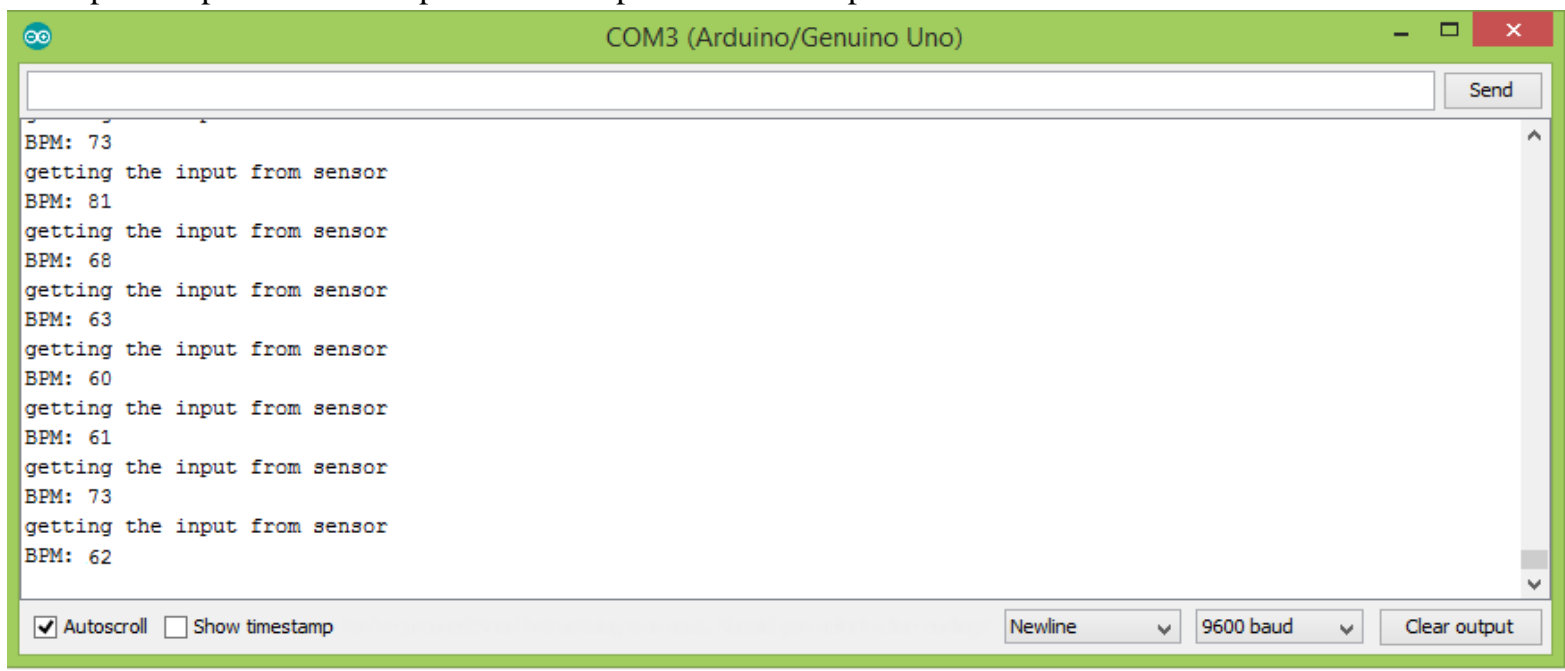




\section{Results on serial Monitor:}

Below Snapshot represents the output that is the pulse rate in terms of graph(Serial plotter) in Adruino IDE.

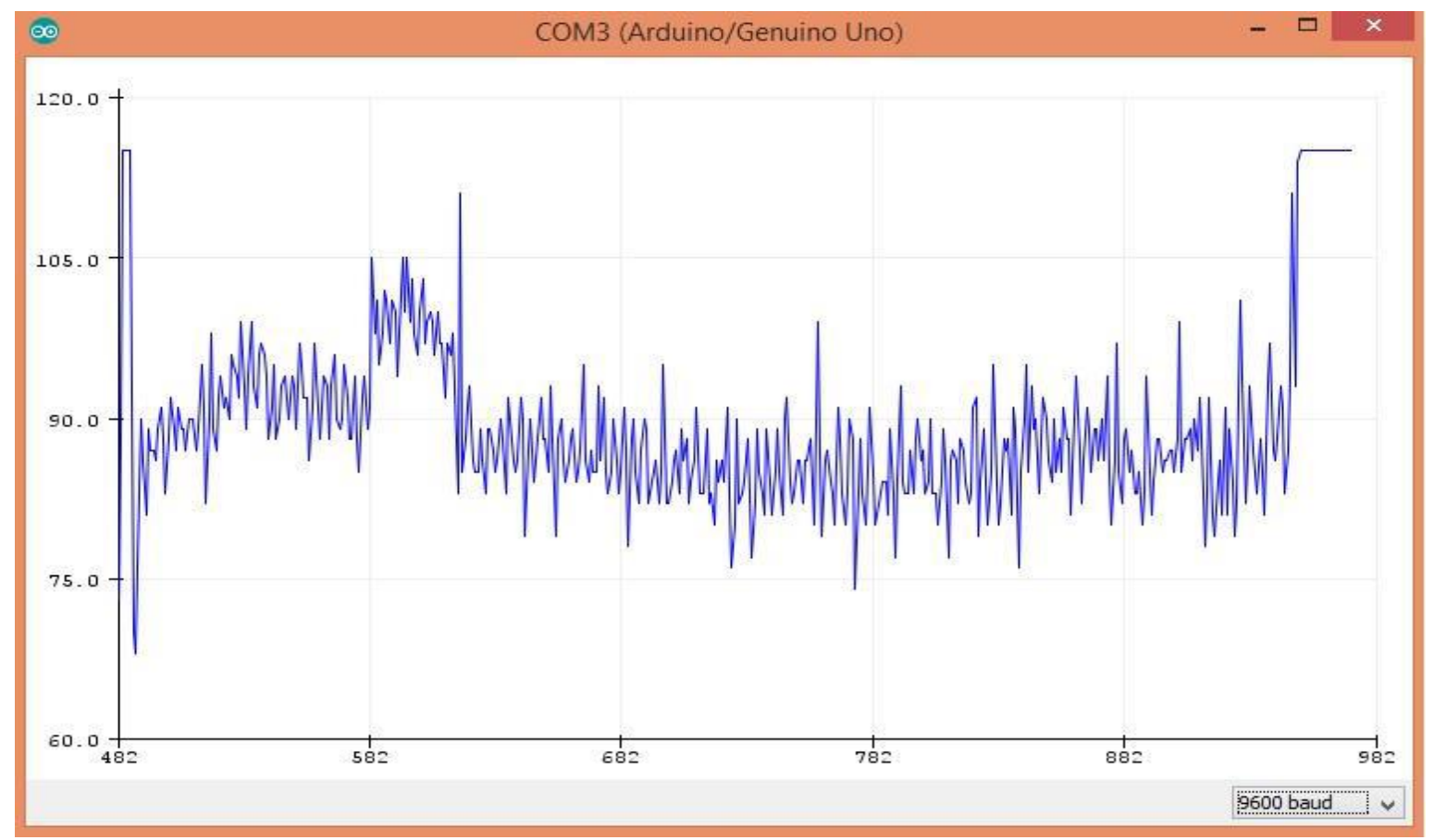

\section{RESULTS ON MOBILE APPLICATION:}

Snapshot 1: below snapshot represents the output that is been displayed on the mobile Screen in the blynk app, The Pulse rate is low when $>40$ and $<60$.

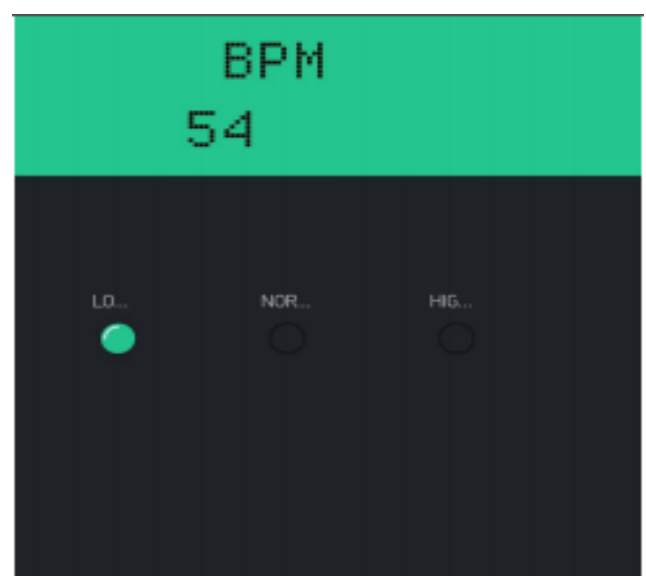

Snapshot 2: below snapshot represents the output that is been displayed on the mobile screen in the blynk app, The Pulse rate is medium when $>60$ and $<100$. 


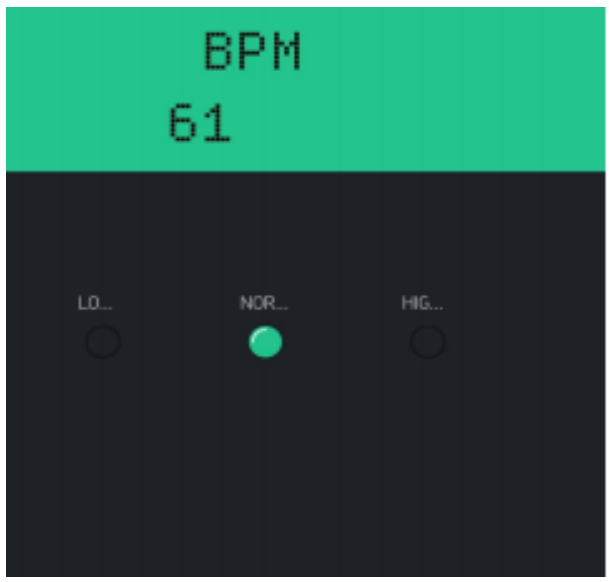

Snapshot 3: below snapshot represents the output that is been displayed on the mobile screen in the blynk app, The Pulse rate is high when $>100$ and $<150$.

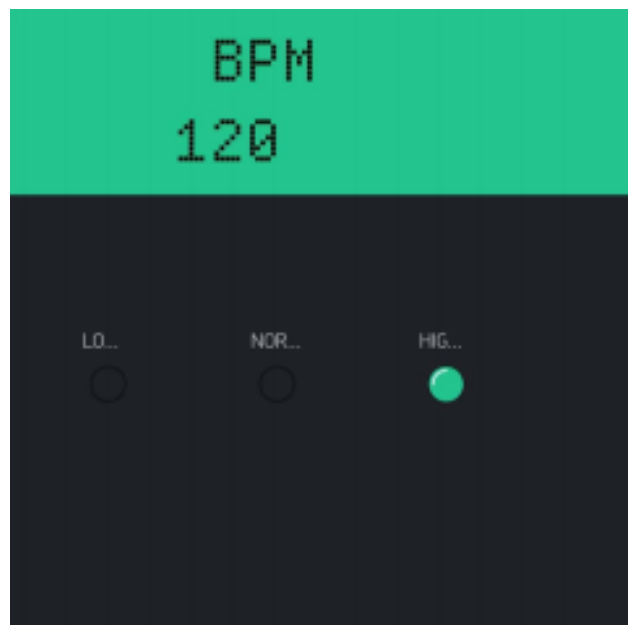

\section{FUTURE WORK}

The current version of the processing application displays the near-time PPG heart rate but does not record anything. Here is a lot of room for improvements. Logging heart rate measurements and PPG samples along with the time-stamp information available from the PC. Beeping sound alarm for heart rates below or above threshold. Heart rate trend over time, etc.

\section{CONCLUSION}

An IoT-based human heartbeat rate monitoring and control system is developed. This system uses the capability of a heart pulse sensor for data acquisition. A human's heartbeat is captured as data signals and processed by the microcontroller. The processed data are transmitted to the IoT platform for further analytics and visualization. Experimental results obtained were found to be accurate as the system was able to sense and read the heartbeat rate of its user and transmits the sensed data via Bluetooth to the Android mobile app(Blynk). From the results obtained, it was found that the heartbeat rate of low if $>40$ and $<60$, medium if $>60$ and $<100$, high if $>100$ and $<150$. Furthermore, this research paper presents an approach that is flexible, reliable, and confidential for a heartbeat rate monitoring and control system using sensor network and
IoT technology. The implemented device can be deployed to the medical field to assist the medical practitioners to efficiently and reliably do their work without difficulties.

\section{REFERENCES}

[1] A microcontroller based automatic heart rate counting system from fingertip Mamun AL, Ahmed N, ALQahtani (JATIT) Journal OF Theory and Applied technology ISSN 1992-8645.

[2] Heartbeat and Temperature Monitoring System for remote patients using Arduino Vikram Singh, R. Parihar, Akash Y TangipahoaD Ganorkar (IJAERS), International Journal of Advanced Engineering and Science eissn2349-6495.

[3] A GSM Enabled Real time simulated Heart Rate Monitoring and control system Sudhindra F, Anna Rao S.J, (IJRET) International Journal of Research In Engineering And Technology, e ISSN 2319-3163.

[4] Heart beat Sensing and Heart Attack Detection Using internet of things: IOT Aboobacker sidheeque, Arith Kumar, K. Sathish,(IJESCE) International Journal Of Engineering Science and Computing, April 2007

[5] A Heartbeat and Temperature Measuring System for Remote Health

[6] Monitoring using Wireless Body Area Network Mohammad Wajih Alam, Tanin Sultana and Mohammad Sami Alam International Journal of Bio Science and Bio-Technology Vol.8, No.1 (2016)

[7] Heartbeat Monitoring Alert via SMS 2009 IEEE Symposium on Industrial Electronics and Applications October 4-6, 2009, Kuala Lumpur, Malaysia. Warsuzarina Mat Jubadi, Siti Faridatul Aisyah 
Mohd Sahak Dept. of Electronics Engineering University Tun Hussein Onn Malaysia Batu Pahat, Johor, Malaysia.

[8] J. Allen, "Photoplthysmography and its application in clinical physiological measurement," Physiol. Meas, vol. 28, pp. R1 R39, 2007.

[9] Wikipedia(2016). Bluetooth.[Online]. Available: https://en.wikipedia.org/wiki/Bluetooth.

[10] T. Tamura, Y. Meada, M. Sekine and M. Yoshida, "Wearable photoplethysmographic sensors - past and present," Electronics, vol. 3 , pp. $282-302,2014$.
[11] Mahmood, N.H.; Uyop, N.; Zulkarnain, N.; Harun, F.K.C.; Kamarudin, M.F.; Linoby, A., "LED indicator for heart rate monitoring system in sport application," Signal Processing and its Applications (CSPA), 2011 IEEE 7th International Colloquium on , vol., no., pp.64,66, 4-6 March 2011.

[12] J. G. Webster, Design of Pulse Oximeters, 1st ed. Bristol: Institute of Physics Publishing, 1997. 